\title{
Numerical investigation of a reaction in a batch reactor: flammability limits
}

\author{
M. J. Sexton* $\quad$ H. S. Sidhu ${ }^{\dagger} \quad$ M. I. Nelson
}

(Received 1 August 2001; revised 18 December 2002)

\begin{abstract}
We study the spatial behaviour of the hydrogen-chlorine reaction in a batch reactor. The solutions exhibit regimes of slow and fast reaction. The main aim of this work is to distinguish between flaming and non-flaming mixtures from the numerical solutions. After setting out a practical criterion for flammability, we construct diagrams in parameter space which display the regions in which the reaction mixture is flammable.
\end{abstract}

\section{Contents}

\section{Introduction}

*Present address: DSTO Pyrmont NSW Australia

†School of Mathematics \& Statistics, University of New South Wales at ADFA, Canberra, Australia. mailto:h.sidhu@adfa.edu.au

$\ddagger$ as above

${ }^{0}$ See http://anziamj. austms.org.au/V44/CTAC2001/Sext for this article,

(C) Austral. Mathematical Soc. 2003. Published 1 April 2003. ISSN 1446-8735 
2 Modelling

C690

3 Numerical method of solution

C692

4 Results

C693

4.1 Three cases . . . . . . . . . . . . . . C695

4.2 Criterion for flammability . . . . . . . . . C697

4.3 Determining ignition boundaries . . . . . . . . C699

5 Discussion

C702

References

C703

\section{Introduction}

This is a preliminary investigation into the behaviour of the reaction between hydrogen and chlorine in a one-dimensional batch reactor. This extends our work on this reaction in a well-stirred semi-batch reactor [8]. In this paper, we are concerned mainly with the issue of distinguishing between a flammable and non-flammable mixture from the numerical solution. Experimentally it is easy to conclude that the mixture is combusting as one would see a flame. How does one distinguish between these two events numerically? In answering this question, we wish to ascertain whether there is a distinct change in the spatial behaviour of the solution between the regimes of fast (flaming) and slow (non-flaming) reaction.

Although gaseous fuels such as methane and propane are commonly referred to as flammable, their mixtures with oxygen or air will only burn if the fuel concentration lie within sharply defined limits: the fuel-lean (lower) and fuel-rich (upper) flammability limits. The most widely used method to determine flammability limits 
is the US Bureau of Mines apparatus [3]. In this method an ignition source is introduced at the lower end of a vertical tube of length $1.5 \mathrm{~m}$. A mixture is deemed to be flammable if a flame can propagate at least half-way up the tube. This criterion was chosen to distinguish between a flammable mixture that is truly capable of propagating a flame indefinitely away from the ignition source and a non-flammable mixture that propagates a flame because the ignition source is 'too strong'. Flammability limits are often investigated by determining the dependence of the flame speed upon the stoichiometry of the reaction mixture. (Here, we define the stoichiometry as being the ratio of the initial pressure of the reactant to the total pressure of the mixture.) Flammability limits are functions of temperature and pressure: the limits widen if the initial temperature is increased or if the pressure is raised. For fixed initial pressure and temperature the limits are dependent upon the precise experimental setup. Lucid introductions to flammability limits are to be found in Glassman [6] and Drysdale [5].

It is therefore of practical interest to determine flammability limits of different reactant mixtures. Hydrocarbon oxidation is kinetically complicated with hundreds of reactions and dozens of chemical species. The study of a smaller reaction mechanism is an important first step in learning to deal with a much larger mechanism. The exothermic reaction considered in this paper is the five-step, fivespecies, hydrogen-chlorine reaction.

We begin in Section 2 by introducing the reaction scheme and the governing equations. The numerical solution method used is the method of lines, described in Section 3, where the governing partial differential equations (PDEs) are discretised in space yielding a system of ordinary differential equations (ODEs). Our numerical results are presented in Section 4 along with diagrams in the control parameter space (initial concentration of hydrogen vs ambient 
temperature, ambient temperature vs length of the reactor and stoichiometric parameter vs length of reactor) showing the boundary between flammable and non-flammable mixtures.

\section{Modelling}

The reaction of hydrogen and chlorine to produce hydrogen chloride

$$
\left[\mathrm{H}_{2}\right]+\left[\mathrm{Cl}_{2}\right] \rightarrow 2[\mathrm{HCl}]
$$

is modelled by the five-step mechanism

$$
\begin{aligned}
{\left[\mathrm{Cl}_{2}\right]+[\mathrm{M}] } & \rightarrow 2[\mathrm{Cl}]+[\mathrm{M}], \\
{\left[\mathrm{H}_{2}\right]+[\mathrm{Cl}] } & \rightarrow[\mathrm{HCl}]+[\mathrm{H}], \\
{[\mathrm{H}]+\left[\mathrm{Cl}_{2}\right] } & \rightarrow[\mathrm{HCl}]+[\mathrm{Cl}], \\
{[\mathrm{H}]+[\mathrm{HCl}] } & \rightarrow\left[\mathrm{H}_{2}\right]+[\mathrm{Cl}], \\
2[\mathrm{Cl}]+[\mathrm{M}] & \rightarrow\left[\mathrm{Cl}_{2}\right]+[\mathrm{M}],
\end{aligned}
$$

as described by Coppersthwaite et al. [2]. The species $[M]$ is a third body. Formally $[M]=\sum_{i=1}^{5}\left[X_{i}\right]$ where $\left[X_{i}\right]$ is the concentration of the $i^{t h}$ species present in the vessel. Following our previous study [8], we simplify the expression for the concentration of the third body by assuming that $[\mathrm{M}]=\left[\mathrm{H}_{2}\right]^{0}+\left[\mathrm{Cl}_{2}\right]^{0}$ where $\left[\mathrm{H}_{2}\right]^{0}$ and $\left[\mathrm{Cl}_{2}\right]^{0}$ represent the initial concentrations of hydrogen and chlorine respectively. This is reasonable as we found that the concentrations for the radicals are of the order of $10^{-5}$ compared to the values of $\left[\mathrm{H}_{2}\right]^{0}$ and $\left[\mathrm{Cl}_{2}\right]^{0}$ which are of order one.

The governing reaction-diffusion equations for the concentrations of the reactants in a one-dimensional reactor of length $L$ (mea- 
sured in metres) are

$$
\begin{aligned}
\frac{\partial\left[H_{2}\right]}{\partial t}= & -k_{3}[C l]\left[H_{2}\right]+k_{5}[H][H C l]+D_{H_{2}} \nabla^{2}\left[H_{2}\right], \\
\frac{\partial\left[C l_{2}\right]}{\partial t}= & -k_{2}\left[C l_{2}\right][M]-k_{4}[H]\left[C l_{2}\right]+k_{6}[C l]^{2}[M] \\
& +D_{C l_{2}} \nabla^{2}\left[C l_{2}\right], \\
\frac{\partial[H]}{\partial t}= & k_{3}[C l]\left[H_{2}\right]-k_{4}[H]\left[C l_{2}\right]-k_{5}[H][H C l] \\
& +D_{H} \nabla^{2}[H], \\
\frac{\partial[C l]}{\partial t}= & 2 k_{2}\left[C l_{2}\right][M]-k_{3}[C l]\left[H_{2}\right]+k_{4}[H]\left[C l_{2}\right] \\
& +k_{5}[H][H C l]-2 k_{6}[C l]^{2}[M]+D_{C l} \nabla^{2}[C l], \\
\frac{\partial[H C l]}{\partial t}= & k_{3}[C l]\left[H_{2}\right]+k_{4}[H]\left[C l_{2}\right]-k_{5}[H][H C l] \\
& +D_{H C l} \nabla^{2}[H C l],
\end{aligned}
$$

where the diffusion of each chemical species is given by the final term in each equation. (The units of each species are $\mathrm{molm}^{-3}$.) Since this is a preliminary investigation, for simplicity, we ignore multi-component diffusion. The rate of reaction step $i$ has a temperature dependence in the form of the Arrhenius equation $k_{i}=$ $A_{i} \exp \left(-E_{i} / R T\right)$ for $i=2-6$ where $A_{i}$ are the pre-exponential factors, $E_{i}$ are the activation energies for reaction steps (2-6), and $R$ is the universal gas constant. The energy equation is

$$
\begin{aligned}
\rho c_{p} \frac{\partial T}{\partial t}= & \left(-\Delta H_{2}\right) k_{2}\left[C l_{2}\right][M]+\left(-\Delta H_{3}\right) k_{3}[C l]\left[H_{2}\right] \\
& +\left(-\Delta H_{4}\right) k_{4}[H]\left[C l_{2}\right]+\left(-\Delta H_{5}\right) k_{5}[H][H C l] \\
& +\left(-\Delta H_{6}\right) k_{6}[C l]^{2}[M]+\kappa \nabla^{2} T
\end{aligned}
$$

where $\Delta H_{i}$, for $i=2-6$ are the enthalpies of reaction step $i, \rho$ is the average initial density, $c_{p}$ is the average initial heat capacity at constant pressure, and $T$ is the temperature within the reactor. 
The first five terms of the RHS of (8) describe the heat of reaction derived from each step of the mechanism, and the conduction of heat is described by the last term. The values for the activation energies, pre-exponential factors and enthalpies are those used by Coppersthwaite et al. [2] and the heat capacities are sourced from Atkins [1]. Since the thermal conductivity of the $[H]$ radical is far greater than any other of the chemical species, we used its value given by [10] as the overall thermal conductivity. The diffusion coefficients used in this investigation are $D_{H_{2}}=3.4 \times 10^{-5} \mathrm{~m}^{2} \mathrm{~s}^{-1}$, $D_{C l_{2}}=3.01 \times 10^{-5} \mathrm{~m}^{2} \mathrm{~s}^{-1}, D_{H}=2 \sqrt{2} D_{H_{2}}, D_{C l}=2 \sqrt{2} D_{C l_{2}}, D_{H C l}=$ $1.246 \times 10^{-5} \mathrm{~m}^{2} \mathrm{~s}^{-1}$.

We shall assume that the initial concentrations of $\left[\mathrm{H}_{2}\right]$ and $\left[\mathrm{Cl}_{2}\right]$ are spatially uniform and that the temperature within the reactor is at ambient temperature. To complete the model formulation, no flux boundary conditions for the chemical species are used at the reactor walls and Dirichlet boundary conditions are imposed on temperature. Therefore, the boundary conditions are

$$
\frac{\partial\left[X_{i}\right]}{\partial x}=0 \quad \text { and } \quad T=T_{a} \quad \text { at } \quad x= \pm L / 2,
$$

where the domain is $x \in[-L / 2, L / 2]$ and $T_{a}$ is the temperature of the reactor walls.

\section{Numerical method of solution}

We implement the method of lines to solve the governing equations (7-8) with boundary conditions (9). The spatial derivatives are discretised, resulting in a system of ODEs which can then be integrated by one of the readily available ODE integrators. The spatial temperature nodal points are given by $T_{j}, j=1, \ldots, n$. Using a 
centred difference discretisation scheme, the heat conduction term in equation (8) becomes

$$
\nabla^{2} T_{j}=\frac{1}{(\Delta x)^{2}}\left(T_{j-1}-2 T_{j}+T_{j+1}\right),
$$

and similarly for the diffusion term in the rate equations for each of the chemical species. The system of six PDEs is now represented by $6 n$ ODEs where $n$ is the number of nodal points used. The no-flux boundary conditions for the chemical species $[X]$ in (9) is calculated by a second-order approximation giving the boundary values as

$$
\begin{aligned}
{[X(1)] } & =\frac{4[X(2)]-[X(3)]}{3}, \\
{[X(n)] } & =\frac{4[X(n-1)]-[X(n-2)]}{3} .
\end{aligned}
$$

Multiple time-scales are common in combustion systems and as a result a stiff integrator is required. For this problem, we used Matlab's stiff solver ode23s. In order to capture the flame front, a value of $n$ must be chosen so that $L /(n-1)$ is of the order of $1 \mathrm{~mm}$ $(0.001 \mathrm{~m})$, thereby indicating that a large number of grid points are required to account for the moving flame.

\section{Results}

As mentioned earlier, the main aim of this work is to determine the transition between the slow and fast reaction zone from the character of the numerical solutions, thus distinguishing between flammable and non-flammable mixtures. In flammability experiments $[5,6$, e.g.] it is common to keep the total pressure of the mixture constant and vary the stoichiometry of the mixture until the transition between a non-flammable and flammable mixture 


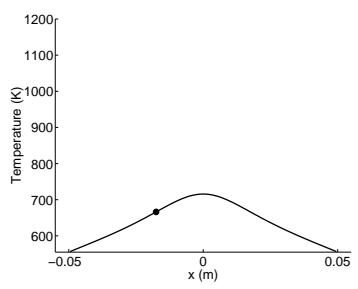

(a) $t=5.02 \mathrm{~s}$

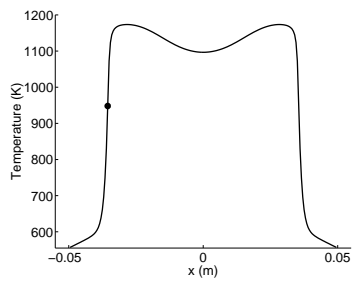

(d) $t=5.12 \mathrm{~s}$

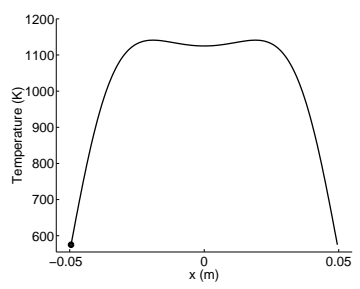

(g) $t=5.26 \mathrm{~s}$

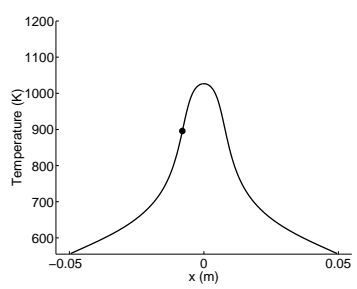

(b) $t=5.08 \mathrm{~s}$

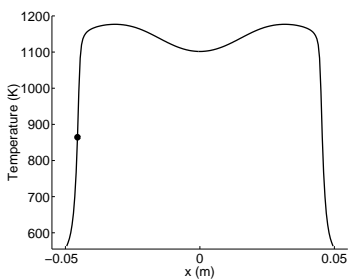

(e) $t=5.14 \mathrm{~s}$

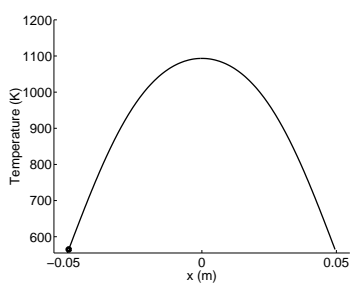

(h) $t=5.60 \mathrm{~s}$

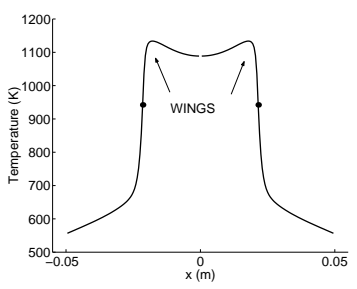

(c) $t=5.10 \mathrm{~s}$

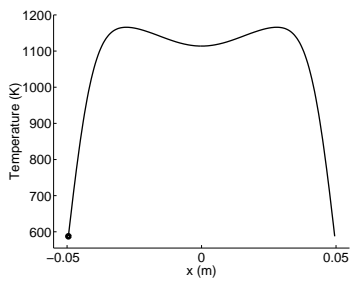

(f) $t=5.19 \mathrm{~s}$

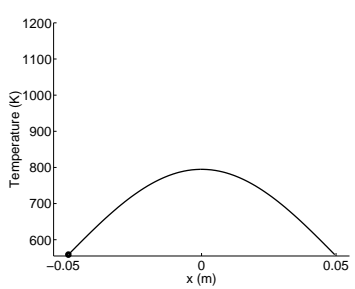

(i) $t=6.88 \mathrm{~s}$

FiguRE 1: Evolution of temperature during the combustion process for $\alpha=0.5$, that is, $\left(\left[\mathrm{H}_{2}\right]^{0},\left[\mathrm{Cl}_{2}\right]^{0}\right)=(1,1) \mathrm{mol} \mathrm{m}^{-3}, L=0.1 \mathrm{~m}$ and $T_{a}=555 \mathrm{~K}$. The position of maximum slope is depicted on each solution curve by a dot. 


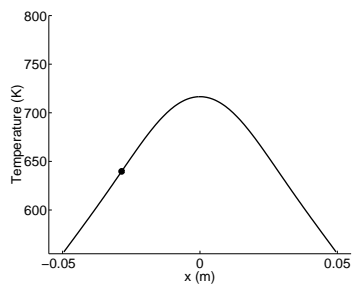

(a) $t=9.8 \mathrm{~s}$

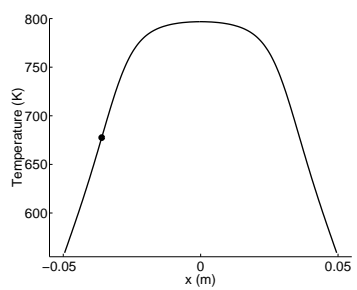

(b) $t=10.29 \mathrm{~s}$

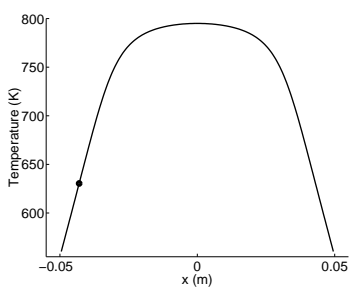

(c) $t=10.43 \mathrm{~s}$

FiguRE 2: Evolution of temperature profiles for $\alpha=0.38$ that is, $\left(\left[\mathrm{H}_{2}\right]^{0},\left[\mathrm{Cl}_{2}\right]^{0}\right)=(0.76,1.24) \mathrm{mol} \mathrm{m}^{-3}$. The rest of the description is as in Figure 1.

is observed. Assuming that the total initial concentration of the mixture is constant, for example, $\left[\mathrm{H}_{2}\right]^{0}+\left[\mathrm{Cl}_{2}\right]^{0}=\mathrm{C}$ we define the parameter $\alpha$ so that $\left[\mathrm{H}_{2}\right]^{0}=\alpha C$ and $\left[\mathrm{Cl}_{2}\right]^{0}=(1-\alpha) C$. Then $\alpha$ is the experimental stoichiometric parameter if one relates pressure to concentration via the ideal gas law. During our numerical investigation we found three distinct types of solution profiles, and we discuss these in more detail in the next section.

\subsection{Three cases}

In this and the next section, we fix $C=2 \mathrm{~mol} \mathrm{~m}^{-3}, T_{a}=555 \mathrm{~K}$, $L=0.1 \mathrm{~m}$ and vary $\alpha$ to determine the different types of spatial behaviour which may be possible for this reaction in the batch reactor.

Figure 1 illustrates the evolution of the temperature profile for case 1 . There is a gradual increase of temperature at the middle of the reactor followed by the formation of a structure which we call 'wings' as shown in Figure 1(c). The presence of these 'wings' indi- 
cates that the maximum temperature is no longer at the middle of the reactor; its location gradually propagates towards the edge of the reactor. As time progresses, the location of maximum temperature returns to the middle of the reactor, as shown in Figure $1(\mathrm{~h})$, and the spatial temperature profile resembles its earlier parabolic shape before finally decaying uniformly to the ambient temperature. For this case, the maximum temperature rise was around $621 \mathrm{~K}$ above ambient.

It is clear then that in case $1 \mathrm{a}$ wave is formed at the centre of the reactor which gradually moves to the edge of the reactor as the 'wings' structure moves out in the manner described above.

Figure 2 illustrates the second type of observed behaviour. Here, unlike the previous case, the maximum temperature is always located at the middle of the reactor and there are no 'wings'. Initially the profile is parabolic as shown in Figure 2(a). Then observe that the profile becomes 'flat' at the middle in Figure 2(b), which we call a 'plateau', and this 'plateau' increases in length as shown in Figure 2(c) and appears to 'propagate' towards the reactor walls. As time progresses, one sees the reverse taking place; the 'plateau' structure decreases in length and the profile goes from Figure 2(c) back to Figure 2(a), regaining its original parabolic shape before decaying to the ambient value. The maximum temperature rise in this case was still significant, reaching approximately $230 \mathrm{~K}$ above the ambient value.

Finally for case 3, shown in Figure 3, the temperature profile is always parabolic and no distinct structures such as 'wings' or a 'plateau' are observed. The temperature profile decays to the ambient value soon after $t=16.55$ seconds as shown in Figure 3(c). The maximum temperature rise was approximately $133 \mathrm{~K}$ above the ambient value. 


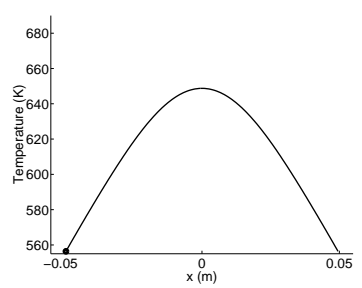

(a) $t=10.7 \mathrm{~s}$

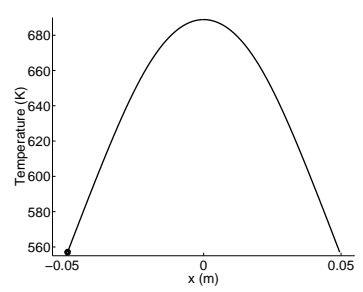

(b) $t=12.54 \mathrm{~s}$

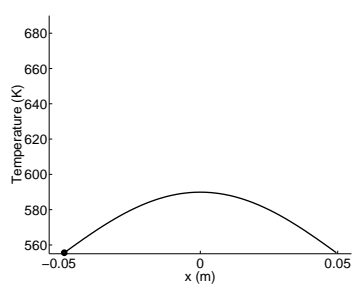

(c) $t=16.55 \mathrm{~s}$

FiguRE 3: Evolution of temperature profiles for $\alpha=0.35$, that is, $\left(\left[\mathrm{H}_{2}\right]^{0},\left[\mathrm{Cl}_{2}\right]^{0}\right)=(0.7,1.3) \mathrm{mol} \mathrm{m}^{-3}$. The rest of the description is as in Figure 1.

\subsection{Criterion for flammability}

Flammability limits are commonly described in the stoichiometryflame speed parameter plane $[5,6]$. In calculating the flame speed, one must determine the variations in spatial behaviour (as a function of time) of a specified quantity. We choose to track the location of maximum temperature (an easily determined value experimentally). To do this, we found the maximum temperature on the nodal points. In calculating the flame speed, we found that the resulting curve was non-smooth (for cases 1 and 2, and case 3 has zero wave speed, as seen in Figure 3). Initially we suspected that the non-smoothness was due to the crude way in which we estimated the location of the maximum temperature, therefore we interpolated the solution data and repeated the calculation, obtaining the same result. Consequently, we abandon this approach for a more tangible criterion, the maximum slope in temperature.

To calculate the maximum slope in temperature, we have used the Matlab inbuilt function diff. We did not interpolate the solution data (we used $n=101$; the solution is calculated every $0.5 \mathrm{~mm}$ 
which is sufficient to assess all relevant behaviour) and therefore, the slope corresponds to nodal values. In calculating the flame speed using this quantity, a similar result was found to the previous case, but with a smoother structure. The reason for this increased accuracy is probably due to the robustness of the solution data to this kind of measure.

We now turn our attention to the position of the maximum slope (in the spatial dimension) for the temperature profiles discussed for the three cases in Section 4.1. These positions are labeled by a bold circle in Figures 1-3. Figure 4(a) and (b) display the evolution of the position of this maximum slope for cases 1 and 3 respectively. Figure 4(a) clearly shows that the location of the maximum slope 'jumps' from the edge of the reactor (at $t=4.927$ seconds) to a point approximately $25 \%$ of the way into the reactor (at $t=4.932$ seconds). Subsequently it proceeds towards the centre of the reactor before turning around and returning towards the wall of the reactor. Similar behaviour was observed in case 2. Repeating this calculation for case 3, we obtain a constant line as shown in Figure 4(b) implying that the position of maximum slope always occurs on the boundary.

Before setting the criterion for a flaming mixture to be the presence of a discontinuity in the location of the maximum slope of the temperature profile, it is essential that we rule out numerical inaccuracy as being the cause of this discontinuity.

The reason for this behaviour is seen by examining Figure 4(c) where the numerical derivative of the temperature profile is shown for case 1 ( Figures 1 and 4(a)) at $t=4.927$ seconds. Observe that the derivative is not monotone. The reason for the 'jump' is the existence of two local maxima (each with almost the same value) in the derivative at $x=-0.05 \mathrm{~m}$ and $-0.028 \mathrm{~m}$. For $t>4.927$ seconds, the local maxima at $x=-0.028 \mathrm{~m}$ is larger than the value at the 


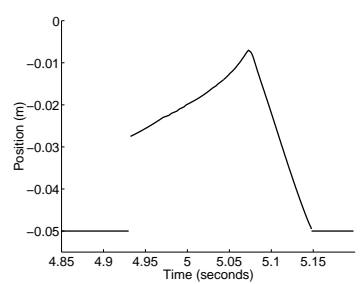

(a) $\alpha=0.5$

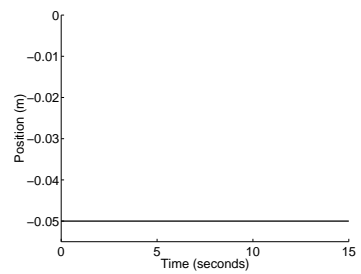

(b) $\alpha=0.35$

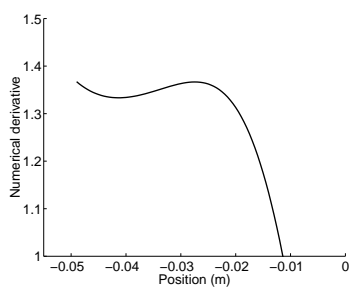

(c)

Figure 4: The position of the maximum slope in temperature during the reaction for (a) $\alpha=0.5$ and (b) $\alpha=0.35$. (c) $\mathrm{Nu}-$ merical derivative of the temperature profile for the case in (a) at $t=4.927$ seconds.

edge of the reactor producing the 'jump' observed in Figure 4(a) from the edge to $x=-0.028 \mathrm{~m}$. Therefore, the discontinuity is not a numerical artifact, rather it describes a distinct change in the nature of the numerical solution. We must also mention that in calculating the maximum slope in temperature, we did not use an interpolation scheme. Using this criterion, we concluded that the mixture is flammable for $0.38<\alpha<0.9$ when $C=2 \mathrm{~mol} \mathrm{~m}^{-3}$, $T_{a}=555 \mathrm{~K}$ and $L=0.1 \mathrm{~m}$.

\subsection{Determining ignition boundaries}

Experimentally it is common to describe ignition in a pressureambient temperature diagram. Figure 5(a) graphs the critical value of $\left[\mathrm{H}_{2}\right]^{0}$ required for flaming combustion for a value of the ambient temperature $T_{a}$ for different reactor sizes using our criterion for flammability described in the previous section. (The pressure of the system is easily calculated from $\left[H_{2}\right]^{0}$ using the ideal gas law.) Flaming/non-flaming combustion is found to the right/left of each 


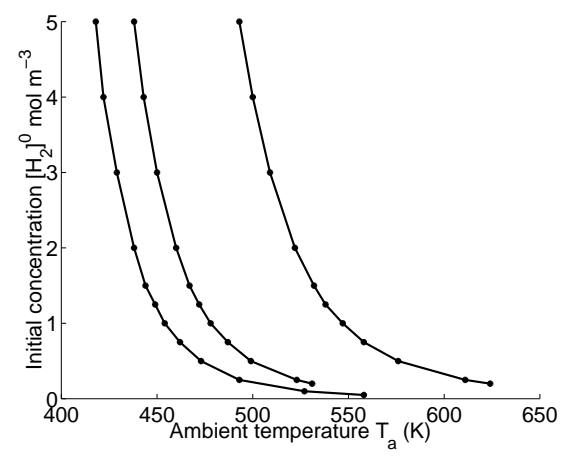

(a) $L=1,0.5$ and $0.1 \mathrm{~m}$, from left to right.

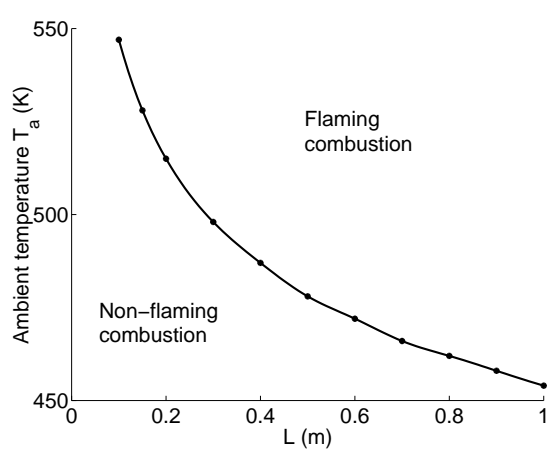

(b)

FiguRE 5: (a) Onset of flaming combustion described by the initial concentration $\left[\mathrm{H}_{2}\right]^{0}$ and ambient temperature $T_{a}$ for different sizes of the reactor $\mathrm{L}$ and $\left[\mathrm{Cl}_{2}\right]^{0}=\left[\mathrm{H}_{2}\right]^{0}$ that is, $\alpha=0.5$. (b) Critical size of the reactor for fixed initial concentration of $\left(\left[\mathrm{H}_{2}\right]^{0},\left[\mathrm{Cl}_{2}\right]^{0}\right)=$ $(1,1) \mathrm{mol} \mathrm{m}^{-3}$ and ambient temperature $T_{a}$.

curve. For a fixed ambient temperature $T_{a}$, Figure $5(\mathrm{a})$ shows how an increasing value of the initial concentration $\left[\mathrm{H}_{2}\right]^{0}$ (and consequently a larger pressure) is required for combustion as the length of the reactor decreases. Figure 5(b) shows how for a fixed initial concentration $\left(\left[\mathrm{H}_{2}\right]^{0},\left[\mathrm{Cl}_{2}\right]^{0}\right)=(1,1) \mathrm{mol} \mathrm{m}^{-3}$, the critical ambient temperature required for flaming combustion reduces as the reactor length increases.

Figure 6(a) shows how the flammability limits depends upon the size of the reactor. Each point corresponds to the last value where the maximum slope in temperature is always found at the wall of the reactor. Therefore, a flame is found within the region bounded by the points. Observe that for a fixed ambient temperature, there exists a critical reactor length, $L_{\text {crit }}$, such that for $L<L_{\text {crit }}$, the 


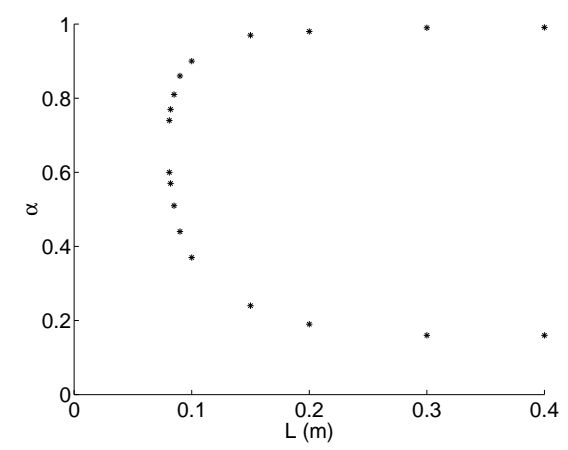

(a)

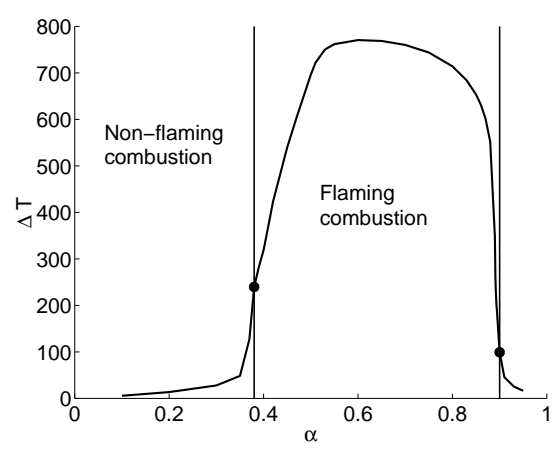

(b)

Figure 6: (a) Flammability limits described in the $(L, \alpha)$ plane with flames existing inside the region for $T_{a}=555 \mathrm{~K}$. (b) Maximum $\Delta T$ versus $\alpha$ for $T_{a}=555 \mathrm{~K}$ and $L=0.1 \mathrm{~m}$. The position of maximum and minimum slope is depicted with a dot.

mixture can never be flammable. This observation is implied by the experimental work described in [3].

Finally, Figure 6(b) graphs the maximum change in temperature versus $\alpha$. The response diagram has a unique solution for any value of $\alpha$ and there is no obvious indication of a flammable region. When the response curve is single-valued, criticality can be defined using the concept of absolute or normalised sensitivity as described by [4, 7]. We define criticality by determining the maximum slope on this curve (absolute sensitivity), and then the range of $\alpha$ for which the mixture is flammable corresponds exactly to the discontinuity criterion discussed earlier. It is encouraging that these definitions of flammability agree. 


\section{Discussion}

In this paper we have investigated a reaction-diffusion model for the reaction of hydrogen with chlorine in a one-dimensional batch reactor. Initially we defined criticality in terms of the position within the reactor at which the maximum temperature gradient is found. We identified a system as subcritical if this position remains at the edge of the reactor during the course of the 'experiment', for example Figure 4(b). For a supercritical system the location changes with time, Figure 3(a). Using this definition, we determined the boundary in the initial pressure-ambient temperature space at which the system changes from subcritical to supercritical, Figure 5(a), and investigated how the critical ambient temperature varies with the reactor length, Figure 5(b).

If we fix the initial pressure and temperature and vary the stoichiometric coefficient $(\alpha)$, then there are two critical limits: a fuellean value and a fuel-rich value. At these values the system changes from subcritical to supercritical, or vice-versa. In Figure 6(a) we showed how these limits vary with the reactor length, establishing that there is a critical reactor length below which any reaction mixture assembled at a specified pressure and initial temperature is non-flammable. We also considered a second definition of criticality based upon the variation in the maximum temperature rise as $\alpha$ is varied. In this method, the transition from subcritical to supercritical is defined in terms of the absolute sensitivity of the response curve to changes in the stoichiometry of the reaction mixture. The values calculated from these two definitions of criticality were in agreement.

It is known experimentally that flammability limits are sharply defined [5]. This feature is not present in our current model. The reason for this is that the model does not include a mechanism for 
heat-loss [9]. If heat-loss is modelled by a term of the form $-\chi T$ on the RHS of equation (8) then there should be a critical value of the heat-loss coefficient $\chi$ at which the unique response curve shown in Figure 6(b) splits into disjoint branches. We intend investigating this point at a later date. In doing so it will be interesting to see if our two definitions of criticality are still in agreement.

Acknowledgements: The authors are very grateful to R. O. Weber and G. N. Mercer for their helpful discussions. M.I.N. is supported by a grant by the Australian Research Council.

\section{References}

[1] P. W. Atkins. Physical Chemistry, pages 816-817. Oxford University Press, third edition, 1986. C692

[2] D. P. Coppersthwaite, J. F. Griffiths and B. F. Gray. Oscillations in the $\mathrm{H}_{2}+\mathrm{Cl}_{2}$ Reaction: Experimental Measurements and Numerical Simulation, J. Phys. Chem, 95, 6961-6967, 1991. C690, C692

[3] H. F. Coward and G. W. Jones. Limits of flammability of gases and vapours. Bulletin - United States Bureau of Mines, 503, 1952. C689, C701

[4] R. M. Chemburkar, M. Morbidelli and A. Varma. Parameter sensitivity of a CSTR. Chem. Engng. Sci., 41, 1647-1654, 1986. C701

[5] D. Drysdale. Limits of flammability and premixed flames. In An Introduction to Fire Dynamics, chapter 3, pages 75-108. 
John Wiley \& Sons, second edition, 1999. C689, C693, C697, C702

[6] I. Glassman. Flammability limits. In Combustion, chapter 4, pages 162-170. Academic Press, third edition, 1996. C689, C693, C697

[7] M. Morbidelli and A. Varma. A Generalized Criterion for Parametric Sensitivity: Application to Thermal Explosion Theory. Chem. Engng. Sci., 43, 91-112, 1988. C701

[8] H. S. Sidhu, M. J. Sexton, M. I. Nelson, G. N. Mercer and R. O. Weber. A simple combustion process in a semi-batch reactor. In Proc. 4th Engng. Maths. Appl. Conf. EMAC 2000 (editors: R. L. May, G. F. Fitz-Gerald and I. H. Grundy), pages 251-254, Institute of Engineers, 2000. C688, C690

[9] D. B. Spalding. A theory of inflammability limits and flame-quenching, Proc. Roy. Soc. Ser. A, 240, 83-100, 1957. C703

[10] WebElements. [Online] http://www.webelements.com/ webelements/elements/text/H/heat.html C692 